
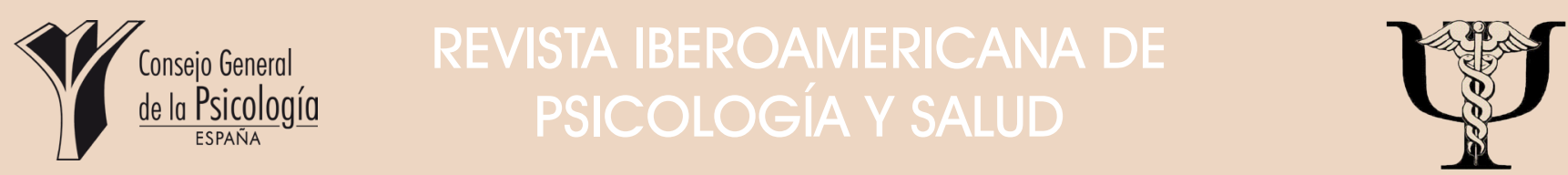

Revista Oficial de la Federación Iberoamericana de Asociaciones de Psicología (FIAP)

[Official Journal of the Latin-American Federation of Psychological Associations]

\title{
Revisión sistemática en el Síndrome del Acento Extranjero: intervención y terapia del lenguaje.
}

\author{
Álvaro Checa-Moreno' y Raúl Quevedo-Blasco²*
}

'Facultad de Psicología y Logopedia. Universidad de Granada, Granada, España.

${ }^{2}$ Centro de Investigación Mente, Cerebro y Comportamiento (CIMCYC). Universidad de Granada, Granada, España.

- Recibido: 21 - Mayo - 2016 - Aceptado: 9 - Junio - 2016

\section{RESUMEN}

El objetivo principal de esta investigación es estudiar la literatura científica referente al tratamiento logopédico o terapéutico del lenguaje en el "Síndrome del Acento Extranjero" (SAE). Se realizó a través de una revisión sistemática de todos los documentos (que tuvieran una extensión superior a una página) obtenidos a través de todas las bases de datos pertenecientes o incorporadas a la "Web of Science" (incluida Medline), ERIC y TESEO, durante el periodo temporal comprendido entre el año 2001 y 2015 (ambos años incluidos). Ocho referencias fueron seleccionadas, de las cuales cinco se analizaron finalmente por cumplir con los criterios de inclusión propuestos. Tras un análisis pormenorizado de las mismas, se presentaron los diferentes tratamientos del lenguaje aplicados o propuestos para pacientes con diagnóstico de SAE. Se concluye que actualmente existe un gran desconocimiento en lo que a la práctica clínica de rehabilitación lingüística se refiere. Esto posibilitará una mayor perspectiva interdisciplinar, así como una nueva óptica de conocimiento, para los profesionales del lenguaje, en algunos de los procesos o técnicas terapéuticas que podrían ayudar a favorecer la recuperación.

PALABRAS CLAVE: Síndrome del Acento Extranjero, Tratamiento logopédico, Terapia del lenguaje, Recuperación, Revisión sistemática.

\section{ABSTRACT}

Systematic review in Foreign Accent Syndrome: intervention and speech therapy. The main objective of this research is to study the scientific literature concerning the treatment in the field of the speech therapy or therapeutic treatment of language in 'Foreign Accent Syndrome' (FAS). It was performed with a systematic review of all the documents (with more than a page of length) available from 2001 to 2015 , both inclusive. These documents were retrieved from all the databases belonging to, or incorporated into, the Web of Science (including Medline), ERIC and TESEO. Eight references were selected, five of them were included because those fulfilling the inclusion criteria proposed. After a detailed analysis of those references, were presented the different treatments of language applied or proposed for the patients diagnosed with FAS. Nowadays we found a great ignorance about what concerned the clinical practice of rehabilitation. This will enable a greater interdisciplinary perspective, as well as a new knowledge point of view, for language professionals in some processes or therapeutic techniques that could help to support the recovery.

KEYWORDS: Foreign Accent Syndrome, Speech therapy, Treatment of language, Recovery, Systematic review.

En la actualidad los estudios teóricos y revisiones bibliográficas son aportaciones importantes debido a la información que proporcionan sobre el problema o trastorno analizado en cuestión. Así se pueden ver estudios

*Correspondencia: Raúl Quevedo-Blasco.

Centro de Investigación Mente, Cerebro y Comportamiento (CIMCYC).

Universidad de Granada. Campus de Cartuja, s/n.

Código Postal: 18011 , Granada (España).

E-mail:rquevedo@ugr.es

(c) 2017 Sociedad Universitaria de Investigación en Psicología y Salud. Publicado por Consejo General de Colegios Oficiales de Psicólogos, España. Este es un artículo Open Access
bajo la CC BY-NC-ND licencia (http://creativecommons.org/licencias/by-nc-nd/4.0/). recientes relacionados con la salud, como por ejemplo sobre trastornos del sueño (e.g., lbañez, Dueñas y Sánchez-Waisen, 2015; QuevedoBlasco, Zych y Buela-Casal, 2014), la motivación (e.g., Viseu, Neves de Jesus, Quevedo-Blasco, Lenuta Rus y Canavarro, 2015), trastornos como la depresión (Cardila et al., 2015; Pérez-Wehbe, Perestelo-Pérez, Bethencourt-Pérez, CuéllarPompa y Peñate-Castro, 2014; Vázquez, Torres, Blanco, Otero y Hermida, 2015) o resultados de la rehabilitación neuropsicológica en la calidad de vida (Guàrdia-Olmos, Peró-Cebollero y Gudayol-Ferré, 2015). 
A pesar de ello, este tipo de estudios no se están desarrollado de forma tan exhaustiva para analizar trastornos del lenguaje poco frecuentes, como es el caso del "Síndrome del Acento Extranjero" (SAE). El SAE, también reseñado como FAS (abreviatura aportada del inglés, Foreign Accent Syndrome), fue descrito por vez primera hace poco más de un siglo por el neurólogo francés Marie (1907). Se trata de un trastorno del lenguaje sobrevenido que altera un patrón de habla sólido, propio de una lengua nativa, de tal modo que el acento de la persona afectada se percibe como extranjero con un dialecto diferente de su idioma de origen (Blumstein y Kurowski, 2006).

A día de hoy, el número de casos publicados sigue siendo bastante restringido, lo cual sugiere una baja incidencia en la población (Van der Scheer, Jonkers y Gilbers, 2014). De hecho, en el periodo temporal comprendido entre los años 1941 y 2009, únicamente, fueron registrados como tal, un total de sesenta casos en países de lengua inglesa (Mariën, Verhoeven, Wackenier, Engelborghs y De Deyn, 2009) y setenta hasta el año 2015 (Baizabal-Carvallo y Jankovic, 2015).

Las principales etiologías que se le atribuyen al SAE, son accidentes cerebrovasculares o lesiones cerebrales traumáticas que afectan a regiones significativas en la producción del habla y del lenguaje expresivo (Jones, Story, Collins, Dejoy y Edwards, 2011 1). A pesar de que algunos autores como Coleman y Gurd (2006) sopesaron un origen neurogénico del SAE, a expensas de futuros estudios concluyentes, Keulen et al. (2016) consideran una variante del SAE como psicógena. En contraposición, Miller, Lowit y O'Sullivan (2006) se postulan a favor de la idea de que los cambios producidos en el discurso del SAE pueden tener la misma base fisiológica que otros trastornos de naturaleza motora con alteraciones en la producción de habla como en la disartria o en la apraxia. En los registros neuroanatómicos focales, a través de las evolucionadas técnicas de neuroimagen actuales, no se han descrito áreas de afectación específicas, sino múltiples lesiones corticales y subcorticales que pueden ser la causa (GonzálezÁlvarez, Parcel-lbars, Ávila y Geffner-Sclarsky, 2003).

Según las aportaciones de Kuschmann y Lowit (2012), la aparición de este nuevo acento en el lenguaje, puede ser explicado por una combinación de alteraciones motoras que ocasionan cambios en elementos suprasegmentales del lenguaje. Entre las principales dificultades, se destacan alteraciones en los elementos articulatorios, prosódicos y gramaticales (Van Borsel, Janssens y Satens, 2005). Por otro lado, los pacientes no muestran déficits a nivel de lenguaje comprensivo u expresivo, además de no manifestar deformaciones fonéticas o falta de fluencia como es observable en las afasias motoras (GonzálezÁlvarez et al., 2003).

La inteligibilidad del habla entre los afectados, correlacionándola a las evidencias anteriormente citadas, no se percibe como alterada. De hecho, en el estudio llevado a cabo por Dankovicová y Hunt (2011), un grupo control de sujetos extranjeros es expuesto frente a uno de pacientes con SAE, y en estos casos los evaluadores acústicos fueron incapaces de discriminar acústicamente diferencias significativas entre ambos grupos. Si bien es cierto, que estos resultados difieren en evidencia ante el estudio de Verhoeven et al. (2013).

En términos generales, la jerga propia del $\mathrm{SAE}$, no es reconocida como patológica por normoyentes (sujetos sin alteración auditiva). Es por ello, que al no existir un impedimento sustancial en el desarrollo de las actividades de la vida diaria de estos pacientes, determinados médicos, suponen que no hay problemas de comunicación (Van der Scheer et al., 2014). Sin embargo, en algunos estudios DiLollo, Scherz y Neimeyer (2014) y Miller (2010) hacen referencia a las consecuencias psicosociales adversas en la vida de los pacientes con SAE.

En este punto, es esencialmente dónde emerge la trascendencia de este estudio y así ofrecer una dimensión interventiva mediada, en gran parte, por clínicos especialistas del lenguaje. De esta forma, se ofrece información sobre la rehabilitación de pacientes con SAE, devolviéndoles a su condición de vida propicia y establecida dentro de la comunidad lingüística a la que pertenecen.

Tal y como sucede en los procesos de intervención de otros trastornos del lenguaje, éstos pueden ser abordados desde múltiples disciplinas sanitarias. El objetivo general, del presente estudio, partiendo del desconocimiento que atañe a los profesionales del lenguaje en el tratamiento de dicho trastorno, fue realizar una 
revisión sistemática (RS) con el fin de recopilar todas aquellas investigaciones que ofreciesen una perspectiva de tratamiento logopédica o basada en la terapia del lenguaje en pacientes diagnosticados con $\mathrm{SAE}$, así como analizar su posible efectividad en el ámbito clínico.

\section{MÉTODO}

\section{- DISEÑO Y PROCEDIMIENTO}

Se trata de una revisión sistemática (RS) interpretativa. El periodo de la búsqueda fue del 25 de febrero al 4 de marzo de 2016. $\mathrm{Se}$ revisaron sistemáticamente, siguiendo los criterios establecidos por Perestelo-Pérez (2012), todos los documentos que tuvieran una extensión superior a una página para su posterior análisis y obtener información de interés para el estudio (no incluyendo, por ejemplo, los resúmenes), publicados desde enero de 2001 hasta diciembre de 2015. Este periodo temporal está justificado principalmente porque en el año 2000 se realizó una revisión en la que se recogen datos relevantes del SAE en años previos (Moen, 2000), aunque no focalizados a intervenciones del lenguaje. Un segundo factor, es que además se constata que los últimos 15 años ha sido un periodo muy productivo en investigaciones sobre esta temática.

Fueron consultadas todas las bases de datos incluidas en la Web of Science (WoS; donde están incluidas Colección principal de WoS, Medline, Scielo Citation Index, BIOSIS Citation Index, BIOSIS Previews, Chinese Science Citation Database, Current Contents Connect, Derwent Innovations Index, $\mathrm{KCl}$ - Korean Journal Database, Russian Science Citation Index y Zoological Record), y para disminuir el sesgo de publicación se incluyeron Education Resources Information Center (ERIC) y TESEO. En lo que a la estrategia de investigación se refiere, se desarrolló una maniobra de búsqueda lo más integradora posible para no sesgar la obtención de resultados y conseguir el mayor número de estudios posibles. A pesar de la escasez de publicaciones en español, se realizaron las búsquedas en inglés y español, limitando los campos de búsqueda al título, el resumen y las palabras clave. La estrategia de búsqueda utilizada fue usando el término general "Foreign Accent Syndrome" y, acotando, con la fórmula
[Speech AND Therap* OR Langua* AND Treatment OR Interven* AND Effects] para obtener cualquier variante de los términos "Therapy; Language; Intervention".

\section{- CRITERIOS DE INCLUSIÓN}

Las publicaciones incluidas en esta RS fueron todo tipo de documentos (artículos, revisiones, tesis, libros, capítulos de libros, proceedings, etc.), delimitados dentro del periodo temporal previamente mencionado (de 2001 a 2015), y que favorecieran o efectuaran algún tipo de tratamiento o terapia del lenguaje en los pacientes diagnosticados con SAE. No se aplicó ningún tipo de restricción con respecto a la edad o la etiología del SAE de los pacientes incluidos en los estudios.

\section{- CRITERIOS DE EXCLUSIÓN}

Fueron excluidos todos aquellos estudios que no aplicaran ningún tipo de procedimiento interventivo de lenguaje y/o que no tuvieran una extensión mínima superior a una página. También, se desecharon todos aquellos trabajos donde las terapias habían estado basadas, en su totalidad, en la suministración de medicación (tratamiento farmacológico).

\section{- PROCESO DE SELECCIÓN}

Tras concluir las búsquedas, se procedió a una fase de selección taxonómica estructurada en tres estadios. En el primero, se actuó por medio de una lectura de los títulos y resúmenes de los resultados obtenidos para seleccionar todos aquellos que cumplían con los criterios de inclusión y exclusión; en el segundo, a través de la lectura comprensiva de todos aquellos estudios que fueron seleccionados en el primer estadio y, finalmente, en el último se escogieron exclusivamente aquellos que cumplían los criterios de inclusión. Para verificar los criterios de selección, se realizó un proceso de revisión por pares abierto.

Con respecto a la evaluación de la calidad de los artículos seleccionados no se realizó, puesto que todos comparten la misma metodología de caso único. 


\section{- RECOLECCIÓN DE DATOS}

Los datos fueron recogidos, a través de un análisis pormenorizado de los artículos seleccionados previamente en el tercer estadio de selección. De cada uno de ellos, se extrajeron los datos de mayor relevancia clínica para cumplir con los objetivos propuestos. Fueron examinadas las siguientes variables: las muestras, las etiologías del SAE, los diseños de casos y se recopiló información relativa a los tratamientos del lenguaje (principalmente en cuanto a sus procedimientos, técnicas y efectos rehabilitadores).

\section{RESULTADOS}

\section{- REFERENCIAS SELECCIONADAS}

Por medio de la estrategia de búsqueda llevada a cabo, se obtuvieron un total de 44 documentos, de los cuales 8 fueron seleccionados por el "título" y el "resumen". El resto, fueron excluidos por no cumplir estrictamente con los criterios de inclusión formulados. El procedimiento de selección queda registrado en la Figura 1.

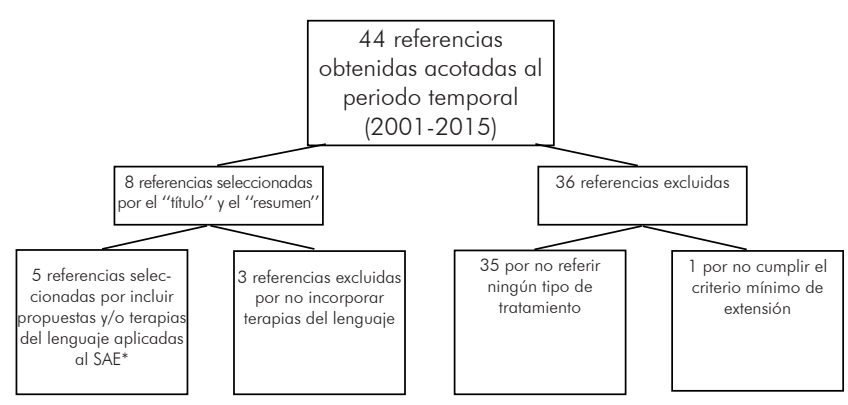

Figura 1. Búsqueda y selección de referencias.

*SAE $=$ Síndrome del Aento Extranjero.

\section{- ANÁLISIS DE CONTENIDO DE LOS ESTUDIOS INCLUIDOS}

Análisis de contenido de los estudios incluidos. Atendiendo a la procedencia nativa de las muestras en los estudios incluidos, se encuentran tres mujeres americanas, una mujer inglesa, una española (que habla catalán y español) y los dos casos restantes no están especificados. Las principales características obtenidas de cada una de las referencias seleccionadas, se describen en la Tabla 1.

\begin{tabular}{|c|c|c|c|c|c|}
\hline Cita & Diseño & Muestra & Etiología del SAE & Terapia del lenguaje & Principales resultados \\
\hline $\begin{array}{l}\text { Liu, Qi, Liu, Liu y Li } \\
\text { (2015) }\end{array}$ & $\begin{array}{l}\text { Caso único; línea base múltiple } \\
\text { (varios pacientes, la misma } \\
\text { intervención). }\end{array}$ & $\begin{array}{l}2 \text { casos clinicos. } \\
\text { Caso I. (C.1.). } \\
\text { Caso 2. (C.2). }\end{array}$ & 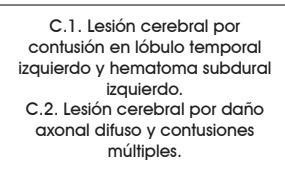 & Aplicada. & $\begin{array}{l}\text { C.1. Recuperación del SAE en } \\
\text { diez diass. } \\
\text { C.2. Recuperación del SAE en } \\
\text { un mes. }\end{array}$ \\
\hline $\begin{array}{l}\text { DiLollo et al. } \\
(2014)\end{array}$ & $\begin{array}{l}\text { Caso único; línea base múltiple } \\
\text { (varios pacientes, la misma } \\
\text { intervención). }\end{array}$ & $\begin{array}{l}2 \text { casos clínicos. } \\
\text { Caso 1. (C.1). } \\
\text { Caso 2. (C.2). }\end{array}$ & $\begin{array}{l}\text { C.1. Lesión traumática severa en } \\
\text { Alteración Temporo-Mandibular } \\
\text { (ATM). } \\
\text { C.2. Prognatismo; Disfunción } \\
\text { de ATM. }\end{array}$ & Propuesta. & $\begin{array}{l}\text { C.1. Tras tres años es capaz de } \\
\text { controlor su acento a pesar de } \\
\text { persistiti involuntartariamente. } \\
\text { C.2. SAE persistente. }\end{array}$ \\
\hline $\begin{array}{l}\text { Moreno-Torres et } \\
\text { al. (2013) }\end{array}$ & $\begin{array}{l}\text { Caso único; línea de base } \\
\text { múltiple (varias conductase, el } \\
\text { mismo paciente). }\end{array}$ & 1 caso clínico. & $\begin{array}{l}\text { Linfadenopatía generalizada } \\
\text { por lesión cervical traumádica } \\
\text { y recurrentes episodios de } \\
\text { migraña. }\end{array}$ & Propuesta. & $\begin{array}{l}\text { No especificados a nivel de } \\
\text { terapia del lenguaje. }\end{array}$ \\
\hline Jones et al. (2011) & $\begin{array}{l}\text { Caso único; línea de base } \\
\text { múltiple (varias conductas, el } \\
\text { mismo paciente). }\end{array}$ & 1 caso clinico. & $\begin{array}{l}\text { Cefalea aguda persistente } \\
\text { localizada en la región } \\
\text { temporo-parietal derecha con } \\
\text { sensitividadd disminuidada en lado } \\
\text { derecho; trastorno del habla y } \\
\text { de la marcha. }\end{array}$ & Aplicada. & $\begin{array}{c}\text { Dificultad para mejorar la } \\
\text { precisión de los sonidos, } \\
\text { inclusoe la la producción de } \\
\text { vocales aisladas con el máximo } \\
\text { modelado. } \\
\text { Alta voluntaria del paciente tras } \\
\text { una sesión. }\end{array}$ \\
\hline $\begin{array}{l}\text { Coughlan, } \\
\text { Lawson y O'Neill } \\
(2004)\end{array}$ & $\begin{array}{l}\text { Caso único; línea de base } \\
\text { múltiple (varias conductas, el } \\
\text { mismo paciente). }\end{array}$ & 1 caso clínico. & $\begin{array}{l}\text { Aparicicín repentina de } \\
\text { hemiparesia derecha, debilidad } \\
\text { facial, disfonía, disfagia y } \\
\text { disartria. }\end{array}$ & Aplicada. & $\begin{array}{l}\text { Tras dos semanas de terapia } \\
\text { intensiva, recupera la fluidez } \\
\text { del habla con un minimo de } \\
\text { disartria. }\end{array}$ \\
\hline
\end{tabular}




\section{- TRATAMIENTOS DEL LENGUAJE APLICADOS}

Las terapias de lenguaje llevadas a cabo en los estudios de Coughlan et al. (2004) y Liu et al. (2015), demostraron evidencias de la eficacia clínica de los tratamientos basados en terapias de fortalecimiento del idioma apoyadas en el ejercicio funcional (Liu et al., 2015) y la terapia del habla y de lenguaje generalizada (Coughlan et al., 2004). En contraposición, no pudo certificarse la validez del programa de tratamiento conductual dirigido a los errores en sonidos de voz (Jones et al., 201 1), por falta de sesiones, consecuencia de la petición de alta voluntaria del paciente durante el tratamiento.

\section{- TRATAMIENTOS DEL LENGUAJE PROPUESTOS}

Aunque no existen evidencias de su efectividad, en dos estudios de la literatura seleccionada, se han propuesto dos terapias de lenguaje frente al SAE a modo de futuras prácticas clínicas. Éstas, han sido fundamentadas en las aportaciones analíticas de dos estudios de caso único tras la evaluación y diagnóstico del SAE. Se exponen, por tanto, una propuesta de intervención basada en la retroalimentación auditiva retardada (Moreno-Torres et al., 2013) y una proposición de terapia del habla y de lenguaje, dirigida hacia las disfluencias o "idioma coaching" (DiLollo et al., 2014).

\section{- PROPUESTA DE INTERVENCIÓN GENERAL EN FUNCIÓN DE LOS ESTUDIOS INCLUIDOS}

Ante la amplia carencia de estudios que incluyan terapias del lenguaje en SAE y, tras analizar la evidencia clínica de éxito en la posible recuperación de estos pacientes a través de algunos de los modelos de tratamiento sugeridos en los artículos de la Tabla 1, a continuación se formula una propuesta general de intervención que asuma las distintas etapas del proceso interventivo, incluyendo el diagnóstico, la evaluación y el tratamiento de este trastorno (Tabla 2).

Propuesta de intervención general en SAE.

\begin{tabular}{|c|c|c|}
\hline Diagnóstico & $\begin{array}{c}\text { Evaluación } \\
\text { (Jones et al., 201 1) }\end{array}$ & Tratamiento \\
\hline $\begin{array}{l}\text { Whitake (1982) propone cuatro criterios para el diagnóstico del SAE: } \\
\text { 1) El acento "extraño" es percibido por el propio paciente, por } \\
\text { conocidos y por el investigador. } \\
\text { 2) El dialecto nativo del paciente es distinto al que tenía antes de } \\
\text { la lesión cerebral. } \\
\text { 3) Está claramente relacionado con daños en el sistema nervioso } \\
\text { central. } \\
\text { 4) No hay ninguna evidencia de que el paciente hablara ninguna } \\
\text { lengua extranjera. } \\
\text { Liu et al. (2015) considera que "el diagnóstico del SAE se basa } \\
\text { fundamentalmente en las manifestaciones clínicas: diferencias de ritmo } \\
\text { ysonido normal pero diferente al acento previo al daño cerebral" (p. 84). } \\
\text { Además, pone de manifiesto la existencia de distintas técnicas de } \\
\text { neuroimagen como DWl, PWl y RM que pueden ayudar a comprender la } \\
\text { reorganización durante la rehabilitación. }\end{array}$ & $\begin{array}{l}\text { Examen motor de voz. } \\
\text {-Entrevista clínica / Anamnesis. } \\
\text { Evaluación del mecanismo de habla. } \\
\text {-Mecanismo respiratorio. Medición de presiones espiratorias e } \\
\text { inspiratorias máximas con medidores de presión (RPM; RPMO1; } \\
\text { Micro-Direct). } \\
\text {-Mecanismo laríngeo. Valorar la presencia o ausencia de disfonía } \\
\text { (No es típica de pacientes con SAE). } \\
\text {-Mecanismo Velofaríngeo. Valoración del funcionamiento } \\
\text { velofaringeo. Hipernasalidad; Hiponasalidad; Otras anomalías de la } \\
\text { resonancia. } \\
\text {-Mecanismo Orofacial. Examen motor oral; Distorsiones; } \\
\text { Sustituciones; Prolongaciones; Omisiones; Inserciones, etc. } \\
\text { Prueba de fuerza con el IOPI lingual. } \\
\text { Prueba articulatoria. } \\
\text {-Prosodia. } \\
\text { Correlación de la tasa de articulación de sílabas por segundo/Velocidad } \\
\text { de articulación. } \\
\text { Entonación. } \\
\text { Ritmo. } \\
\text {-Discurso. }\end{array}$ & $\begin{array}{l}\text { Terapia de fortalecimiento del idioma basada en el ejercicio funcional } \\
\text { (Liu et al., 2015). }\end{array}$ \\
\hline
\end{tabular}

\section{DISCUSIÓN Y CONCLUSIONES}

Actualmente, no existe una amplia cantidad de trabajos sobre las terapias de lenguaje que pueden ofrecerse a pacientes con SAE. Según Moreno-Torres et al. (2013), "No hay informes de tratamientos basados en la teoría..." (p. 521).
A través de este estudio, ha sido posible reunir esa ínfima parte de literatura existente dedicada, en cierto modo, al proceso de intervención en este trastorno, aunque por el contrario si es objeto de análisis la funcionalidad 
del lenguaje en procesos terapéuticos (ViruesOrtega y Froián-Parga, 2015). De esta forma, en términos generales, se suple la carencia de una actuación clínica global y multidisciplinar en casos de SAE diagnosticados.

La presente revisión sistemática pone de manifiesto que, a pesar de que la gran mayoría de los estudios de SAE (revisiones y artículos) están enfocados a discernir la razón y causas de este trastorno, empiezan a emerger nuevas formas de abordaje desde una perspectiva rehabilitadora de una de las enfermedades calificadas como "raras" y que, a pesar de no afectar de forma directa a la inteligibilidad o capacidad comunicativa de estos pacientes, sí la condiciona (DiLollo et al., 2014; Miller, 2010). Por ello, es significativo no prescindir de estos resultados y reiterar la importancia de este estudio en sus implicaciones clínicas. A diferencia del estudio de revisión de Moen (2000), en el cual se analizan las principales causas etiológicas así como las características de voz fonético-fonológicas de los pacientes con SAE, en este estudio se demuestra la efectividad práctica de los tratamientos del habla y del lenguaje, posibilitando la recuperación de estos pacientes y devolviéndoles a "su acento natural".

Pese a que no era un objetivo principal de este estudio, en esta RS se ofrece una propuesta de intervención general en SAE, dirigida especialmente a los profesionales sanitarios destinados al abordaje de los trastornos del habla y del lenguaje, Logopedas, Speech Therapists, Fonoaudiólogos, Ortofonistas, para servir a modo de protocolo base en el proceso de intervención. En ella se ofrece un programa general, en la línea de abordaje clínico, que pretende cubrir las necesidades diagnósticas, evaluativas y terapéuticas del SAE. Si bien es cierto, tal y como comenta Moreno-Torres et al. (2013) "el SAE es un trastorno heterogéneo; sin embargo, puede ser recomendable aplicar un único método de estudio de casos y estrategias adoptadas individualmente". (p. 521). Por ello, dicha propuesta debería quedar sujeta a modificaciones particulares que se ajusten a la realidad propia del paciente diagnosticado.

No obstante, cabe destacar que, aunque la etiología no se ha tenido en cuenta en este estudio como una variable estrictamente determinante en la recuperación del SAE, sería conveniente iniciar próximas líneas de investigación orientadas a comprobar la eficacia de distintas terapias en función del origen, lesiones cerebrales traumáticas, daño cerebral sobrevenido, afecciones traumáticas o genéticas en la Alteración Temporo-Mandibular (ATM). En este estudio se entiende el SAE desde una dimensión rehabilitadora global y considerándolo como una alteración motora con daño neurológico, sin entrar en especificaciones - subcategorías sindrómicas como detallan Keulen et al. (2016) diferenciando un SAE de origen psicógeno. En última instancia, también sigue existiendo la necesidad de realizar futuros estudios que revelen y comparen la efectividad de los tratamientos propuestos (DiLollo et al., 2014; Moreno-Torres et al., 2013) para desarrollar e incrementar posteriores programas de tratamiento.

La valoración de los diversos trastornos mediante revisiones sistemáticas y el análisis de las revistas científicas (e.g., García-Pereira y Quevedo-Blasco, 2015; Velasco, Vilariño, Amado y Fariña, 2014) son estudios que están proliferando en los últimos años y proporcionan información de gran utilidad para el ámbito científico, a pesar de existir diversas limitaciones, como por ejemplo, posibles sesgos de publicación en los estudios analizados. Por eso, podría considerarse la realización de RS futuras que abarquen un mayor número de bases de datos de diversa índole (analizando tanto aquellas en las que se publican los mejores resultados, como otras donde los resultados de las investigaciones no son tan excelentes clínica y estadísticamente). Ofrecer resultados sistematizados de los diferentes problemas de salud y de las intervenciones (aplicadas o propuestas), son resultados de gran interés. Todo esto es relevante para el avance tanto del ámbito científico, como clínico.

\section{- Conflicto de intereses}

Los autores declaran no tener ningún conflicto de intereses.

\section{REFERENCIAS}

Baizabal-Carvallo, J.F., y Jankovic, J. (2015). Speech and voice disorders in patients with psychogenic movement disorders. Journal of Neurology, 262 (11), 2420-2424.

Blumstein, S.E., y Kurowski, K. (2006). The foreign accent syndrome: a perspective. Journal of 
Neurolinguistics, 19, 346-355.

Cardila, F., Martos, A., Barragán, A.B., Pérez-Fuentes, M.C., Molero, M.M., y Gázquez, J.J. (2015). Prevalencia de la depresión en España: Análisis de los últimos 15 años. European Journal of Investigation in Health, Psychology and Education, 5 (2), 267-279.

Coleman, J., y Gurd, J. (2006). Introduction to the theme issue on foreign accent syndrome. Journal of Neurolinguistics, 19, 341-345.

Coughlan, T., Lawson, S., y $\mathrm{O}^{\prime}$ Neill, D. (2004). French without tears? Foreign accent syndrome. Journal of the Royal Society of Medicine, 97 (5), 242-243.

Dankovicová, J., y Hunt, C. (2011). Perception of foreign accent syndrome speech and its relation to segmental characteristics. Clinical Linguistics and Phonetics, 25, 85-120.

DiLollo, A., Scherz, J., y Neimeyer, R.A. (2014). Psychosocial Implications of Foreign Accent Syndrome: Two Case Examples. Journal of Constructivist Psychology, $27(1)$, 14-30.

García-Pereira, S., y Quevedo-Blasco, R. (2015). Análisis de las revistas iberoamericanas de Psicología y de Educación indexadas en el Journal Citation Reports del 2013. European Journal of Education and Psychology, 8, 85-96.

González-Álvarez, J., Parcel-lbars, M.A., Ávila, C., y Geffner-Sclarsky, D. (2003). Una rara alteración del habla de origen neurológico: el síndrome del acento extranjero. Revista de Neurología, 36 (3), 227-234.

Guàrdia-Olmos, J., Peró-Cebollero, M., y GudayolFerré, E. (2015). Neuropsychological rehabilitation and quality of life: A meta-analysis Revista Iberoamericana de Psicología y Salud, 6, 11-18.

lbañez, C., Dueñas, D.R., y Sánchez-Waisen, M.R. (2015). Prevalencia del insomnio en ancianos residenciados y prescripción farmacológica. European Journal of Investigation in Health, Psychology and Education, 5 (1), 5-12.

Jones, H.N., Story, T.J., Collins, T.A., Dejoy, D., y Edwards, C.L. (2011). Multidisciplinary assessment and diagnosis of conversion disorder in a patient with foreign accent syndrome. Behavioural Neurology, 24 (3), 245-255.

Keulen, S., Verhoeven, J., De Witte, E., De Page, L., Bastiaanse, R., y Mariën, P. (2016). Foreign Accent Syndrome As a Psychogenic Disorder: A Review. Frontiers in Human Neuroscience, 10, (168), $1-16$.

Kuschmann, A., y Lowit, A. (2012). Phonological and phonetic marking of information status in Foreign Accent Syndrome. International Journal of Language and Communication Disorders, 46 (2), 156-168.

Liv, H.E. Qi, P., Liu, Y.L., Liv, H.X., y Li, G. (2015).
Foreign accent síndrome: two case reports and literatura review. European Review for Medical and Pharmacological Sciences, 19 (1), 81-85.

Marie, P. (1907). Présentation de molades atteints d'anarthrie par lésion de l'hémisphère gauche du cerveau. Bulletins Et Mémories Société Médicale Des Hôpitaux De Paris, 1, 158-160.

Mariën, P., Verhoeven, J., Wackenier, P., Engelborghs, S., y De Deyn, P.P. (2009). Foreign Accent Syndrome as a developmental motor speech disorders. Cortex, 45 (7), 870-878.

Miller, N. (2010). Foreign accent syndrome-Between two worlds, at home in neither. En D. Watt y C. Llamas (Eds.), Language and identities (pp. 67-75). Edinburgh: Edinburgh University Press.

Miller, N., Lowit, A., y O'Sullivan, H. (2006). What makes acquired foreign accent syndrome foreigns? Journal of Neurolinguistics, 19, 385-409.

Moen, I. (2000). Foreign Accent Syndrome: A review of contemporary explanations. Aphasiology, 74 (1), 5-15.

Moreno-Torres, I., Berthier, M.L., Cid, M.D., Green, C., Gutierrez, A., García-Casares, N., Walsh, S.F., Nabrozidis, A., Sidorava, J., Davila, G., y Carnero-Pardo, C. (2013). Foreign accent syndrome: A multimodal evaluation in the search of neurosciencedriven treatments. Neuropsychologia, 51 (3), 520-537.

Perestelo-Pérez, L. (2012). Standards on how to develop and report systematic reviews in Psychology and Health. International Journal of Clinical and Health Psychology, 13, 49-57.

Pérez-Wehbe, A.I., Perestelo-Pérez, L., BethencourtPérez, J.M., Cuéllar-Pompa, L., y Peñate-Castro, W. (2014). Treatment-resistant depression: A systematic review of systematic reviews. International Journal of Clinical and Health Psychology, 14, 145-153.

Quevedo-Blasco, R., Zych, I., y Buela-Casal, G. (2014). Sleep apnea through journal articles included in the Web of Science in the first decade of the $21 \mathrm{st}$ century. Revista Iberoamericana de Psicología y Salud, 5, 39-53.

Van Borsel, J., Janssens, L., y Satens, P. (2005). Foreign accent syndrome: An organic disorder? Journal of Communication Disorders, 38 (6), 421-429.

Van der Scheer, F., Jonkers, R., y Gilbers, D. (2014). Foreign accent syndrome and force of articulation. Aphasiology, 28 (4), 471-489.

Vázquez, F. L. Torres, A., Blanco, V., Otero, P. y Hermida, E. (2015). Intervenciones psicológicas administradas por teléfono para la depresión: una revisión sistemática y meta-análisis. Revista Iberoamericana de Psicología y Salud, 6, 39-52.

Velasco, J., Vilariño, M., Amado, B. G., y Fariña, F. 
(2014). Análisis bibliométrico de la investigación española en psicología desde una perspectiva de género. Revista Iberoamericana de Psicología y Salud, 5, 105-118.

Verhoeven, J., De Pauw, G., Pettinato, M., Hirson, A. Van Borsel, J., y Marien, P. (2013). Accent attribution in speakers with Foreign Accent Syndrome. Journal of Communication Disorders, 46 (2), 156-168.

Virues-Ortega, J., y Froián-Parga, M.X. (2015). A translational approach to the functional analysis of language in psychotherapy. International Journal of Clinical and Health Psychology, 15, 69-76.
Viseu, J., Neves de Jesus, S., Quevedo-Blasco, R., Lenuta Rus, C., y Canavarro, J.M. (2015). Motivação docente: estudo bibliométrico da relação com variáveis individuais, organizacionais e atitudes laboráis. Revista Latinoamericana de Psicología, 47, 58-65.

Whitaker, H.A. (1982). Levels of impairment in disorders of speech. En R.N. Malatesha y L.C. Hartlage (Eds.), Neuropsychology and cognition (Vol. 1, pp. 168-207). Princeton, New Jersey: Nato Advanced Study Institutes Series D, No.9. 